\title{
Convergence of Discretized Stochastic (Interest Rate) Processes with Stochastic Drift Term.
}

\author{
G. Deelstra* $\quad$ F. Delbaen**
}

* Free University of Brussels V.U.B., Department of Mathematics, Pleinlaan 2, B-1050 Brussels, Belgium tel: 003226293473 and 026293471 fax: 003226293495

e-mail: gdeelstr@dwis1.vub.ac.be

** ETH Zürich, Department of Mathematics, CH - 8092 Zürich, Switzerland e-mail: delbaen@math.ethz.ch Part of this work was done while the second author was full professor at the V.U.B. 


\section{SUMMARY}

For applications in finance, we study the stochastic differential equation $d X_{s}=$ $\left(2 \beta X_{s}+\delta_{s}\right) d s+g\left(X_{s}\right) d B_{s}$ with $\beta$ a negative real number, $g$ a continuous function vanishing at zero which satisfies a Hölder condition and $\delta$ a measurable and adapted stochastic process such that $\int_{0}^{t} \delta_{u} d u<\infty$ a.e. for all $t \in \mathbb{R}^{+}$and which may have a random correlation with the process $X$ itself. In this paper, we concentrate on the Euler discretization scheme for such processes and we study the convergence in $L^{1}$-supnorm and in $\mathcal{H}^{1}$-norm towards the solution of the stochastic differential equation with stochastic drift term. We also check the order of strong convergence.

KEY WORDS Stochastic differential equation stochastic drift term Hölder condition Euler discretization scheme strong convergence

\section{INTRODUCTION}

\subsection{Aim of the present study}

Modeling interest rate fluctuations is one of the major concerns of both practitioners and academics. Among the many models which have been put forward to explain the behavior of the short-term riskless interest rate, there is the famous model of Cox, Ingersoll and Ross ${ }^{1}$. Cox, Ingersoll and Ross ${ }^{1}$ express the interest rate dynamics by the stochastic differential equation

$$
d r_{t}=\kappa\left(\gamma-r_{t}\right) d t+\sigma \sqrt{r_{t}} d B_{t} \quad \forall t \in \mathbb{R}^{+}
$$

with $\kappa, \gamma$ and $\sigma$ strictly positive constants and $\left(B_{t}\right)_{t \geq 0}$ a Brownian motion. In this model, the short-term interest rates are elastically pulled towards the long-term constant value $\gamma$. In Deelstra-Delbaen ${ }^{2,3}$, we have extended the CoxIngersoll-Ross model by allowing a stochastic reversion level $\left(\gamma_{s}\right)_{s \geq 0}$ and by taking the volatility term more general.

In this paper, we consider the stochastic processes $X$ which are introduced in Deelstra-Delbaen ${ }^{2}$ for the purpose of modeling interest rates and which are defined by the stochastic differential equation

$$
d X_{s}=\left(2 \beta X_{s}+\delta_{s}\right) d s+g\left(X_{s}\right) d B_{s} \quad \forall s \in \mathbb{R}^{+}
$$

with $X_{0} \geq 0, \beta \leq 0$, where $g$ is a function vanishing at zero which satisfies the Hölder condition

$$
|g(x)-g(y)| \leq b \sqrt{|x-y|}
$$

with $b$ a constant (or where $g$ is defined by $|g(x)|=k x^{\alpha}$ for $0.5 \leq \alpha \leq 1$ ); and with $\delta: \Omega \times \mathbb{R}^{+} \rightarrow \mathbb{R}^{+}$a measurable and adapted stochastic process such that $\int_{0}^{t} \delta_{u} d u<\infty$ a.e. for all $t \in \mathbb{R}^{+}$. 
The main benefit of these interest rate processes is that we can treat two-factor interest rate models without making assumptions about the correlationbetween the two factors, namely the instantaneous interest rate proce ss $X$ and the stochastic reversion level process $\delta$. We stress this fact because it is not trivial. Most authors of two-factor interest rate models require, for technical reasons, that the factors are uncorrelated or have a deterministic and fixed correlation.

As equation (2) is a Doléans-Dade and Protter's equation, it is shown by $\operatorname{Jacod}^{4}$ that there exists a unique strong solution. Extending comparison results as in Karatzas-Shreve ${ }^{5}$ (p. 293) or Revuz-Yor ${ }^{6}$ (p. 375), it is easy to check that the solution remains positive a.s. (see e.g. Deelstra ${ }^{7}$ ).

In this paper, we discuss the Euler discretization scheme for the stochastic differential equation (2) with a drift term which may depend on a stochastic process with random correlation. In the literature, most papers about approximations of solutions to a stochastic differential equation, do not treat the more general case of a random drift term. In Gihman-Skorohod ${ }^{8}$ and Bally ${ }^{9,10}$, this case is treated but a Lipschitz condition is needed for the diffusion function $g$.

Using results of Kurtz-Protter ${ }^{11}$, we show that the approximating solution converges in $L^{1}$-supnorm towards the solution of the stochastic differential equation (2) and we remark that the convergence also holds in the $\mathcal{H}^{1}$-norm (see e.g. Protter $\left.{ }^{12,13}\right)$. Using Yamada's ${ }^{14}$ method, we check the order of strong convergence.

\subsection{Motivation of strong convergence}

We concentrate on strong convergence results because pathwise approximation is required in different fields: e.g. in control theory and filtering problems, in direct simulation and in testing statistical estimators. For instance, Bucy and Joseph $^{15}$ claim that almost sure stability in filtering problems is practically the only interesting type, for it describes the behavior of the physical realizations of the process. Concepts such as stability in the mean or in probability do not give information about the individual sample functions.

In general, pathwise approximations are necessary when one is interested in the sample paths itself. For instance, what one observes at the exchange are the paths of the prices and not the distributions. But if interest focuses on approximating the moments or the expectations of functionals of the Itô process, then one could better use weak convergence results (see e.g. Kloeden-Platen ${ }^{16}$ ). This is the case for some contingent claims but not for all since practically all known weak convergence results involve contingent claims that are required to be smooth functions of the final stock price alone. For path-dependent derivative assets such as American options or Asian options, the situation is more difficult and results typically do not follow from direct weak convergence arguments.

Besides, as explained in Cutland-Kopp-Willinger ${ }^{17}$, weak convergence has proved to be "weak" in the sense that, for example, convergence of contingent claims does neither imply nor is implied by the convergence of the corresponding replicating trading strategies but has to be proved separately. 


\subsection{Outline of the paper}

In the following section, we state the Euler scheme for the stochastic differential equation (2) with stochastic drift term. We first use results of Kurtz and Protter to prove that this scheme converges in $L^{1}$-supnorm towards the solution and we remark that the convergence also holds in $\mathcal{H}^{1}$-norm. In the last section, we make some comments on the order of strong convergence.

Without further notice, we assume that the filtration $\left(\mathcal{F}_{t}\right)_{t \geq 0}$ satisfies the usual assumptions with respect to $\mathbb{P}$, a fixed probability on the sigma-algebra $\mathcal{F}_{\infty}=\bigvee_{t \geq 0} \mathcal{F}_{t}$. Also $B$ is a continuous process that is a Brownian motion with respect to $\left(\mathcal{F}_{t}\right)_{t \geq 0}$.

\section{THE EULER SCHEME WITH STOCHASTIC DRIFT TERM}

\subsection{The scheme and notations}

We resume with the discretization technique which is known as the Euler scheme. For each $n \geq 1$, we take a subdivision of the interval $[0, T]$

$$
0=t_{0}^{n}<t_{1}^{n}<\cdots<t_{N_{n}}^{n}=T
$$

which does not have to be equidistant. We denote this net by $\triangle_{n}$. For notational use, we drop the index $n$ of the discretization times and we write $N$ in stead of $N_{n}$. The mesh of the net is defined as $\left\|\triangle_{n}\right\|=\sup _{1<k<N}\left|t_{k}-t_{k-1}\right|$. We are working with a sequence of nets $\left(\triangle_{n}\right)_{n}$ with the meshes tending to zero. There is no need to suppose that $\triangle_{n} \subset \triangle_{n+1}$.

Although the solution of the stochastic differential equation (2), namely

$$
d X_{s}=\left(2 \beta X_{s}+\delta_{s}\right) d s+g\left(X_{s}\right) d B_{s} \quad \forall s \in \mathbb{R}^{+}
$$

with $X_{0} \geq 0, \beta \leq 0$, and with $g$ and $\delta$ satisfying some hypotheses, remains nonnegative, the Euler approximations may take negative values. Therefore, we put $g^{\prime}(x)=g\left(x \mathbb{1}_{(x \geq 0)}\right)$. It follows that $g^{\prime}$ also satisfies $\left|g^{\prime}(x)-g^{\prime}(y)\right| \leq b \sqrt{|x-y|}$.

Working with the net $\triangle_{n}$, we look at $X_{\triangle_{n}}(t)$, which we denote by $X_{n}(t)$. For $t$ taken between two netpoints, e.g. $t_{k} \leq t \leq t_{k+1}$ with $k=0, \cdots, N-1$, we define $X_{n}(t)$ as follows:

$$
X_{n}(t)=X_{n}\left(t_{k}\right)+2 \beta X_{n}\left(t_{k}\right)\left(t-t_{k}\right)+\delta\left(t_{k}\right)\left(t-t_{k}\right)+g^{\prime}\left(X_{n}\left(t_{k}\right)\right)\left(B_{t}-B_{t_{k}}\right) .
$$

If we introduce the notation $\eta_{n}(t)=t_{k}$ for $t_{k} \leq t<t_{k+1}$, the terms telescope and we can write

$$
X_{n}(t)=X_{0}+\int_{0}^{t} 2 \beta X_{n}\left(\eta_{n}(u)\right) d u+\int_{0}^{t} \delta\left(\eta_{n}(u)\right) d u+\int_{0}^{t} g^{\prime}\left(X_{n}\left(\eta_{n}(u)\right)\right) d B_{u}
$$




\subsection{Convergence in $L^{1}$-supnorm}

We now turn to the proof that this Euler discretization scheme converges in $L^{1}$-supnorm. This result is based on two papers of Kurtz-Protter ${ }^{11,18}$.

\section{Theorem}

Suppose that the stochastic process $X: \Omega \times \mathbb{R}^{+} \rightarrow \mathbb{R}^{+}$is defined by the stochastic differential equation

$$
d X_{s}=\left(2 \beta X_{s}+\delta_{s}\right) d s+g\left(X_{s}\right) d B_{s} \quad \forall s \in \mathbb{R}^{+}
$$

with $X_{0} \geq 0, \beta \leq 0$ and $g: \mathbb{R} \rightarrow \mathbb{R}^{+}$a function, vanishing at zero and such that there is a constant $b$ with $|g(x)-g(y)| \leq b \sqrt{|x-y|}$.

The measurable and adapted process $\delta: \Omega \times \mathbb{R}^{+} \rightarrow \mathbb{R}^{+}$is assumed to satisfy $\sup _{u} \delta(u, \omega) \in L^{1}$ for all $\omega \in \Omega$.

Under these conditions, the discrete recursive scheme

$$
X_{n}(t)=X_{n}\left(t_{k}\right)+2 \beta X_{n}\left(t_{k}\right)\left(t-t_{k}\right)+\delta\left(t_{k}\right)\left(t-t_{k}\right)+g^{\prime}\left(X_{n}\left(t_{k}\right)\right)\left(B_{t}-B_{t_{k}}\right)
$$

with $t_{k} \leq t \leq t_{k+1}, k=0, \cdots, N-1$, converges in $L^{1}$-supnorm towards the solution of the stochastic differential equation.

\section{Proof}

To begin with, we fix a time $T$ and we consider the case $\mathbb{E}\left[\left(\int_{0}^{T} \delta_{u} d u\right)^{2}\right]<\infty$.

We define the sequence $\left(\sigma_{h}\right)_{h \geq 1}$ by

$$
\sigma_{h}=\inf \left\{t \mid X_{t} \geq h\right\}
$$

and we denote $X_{u} \mathbb{1}_{\llbracket 0, \sigma_{h} \rrbracket}$ by $X_{u}^{(h)}$. Using the equivalent of theorem 3.3 of KurtzProtter $^{11}$ in case of a stochastic drift term (which follows along the same lines and from the results of Kurtz-Protter ${ }^{18}$ ), one sees that the Euler discretization with $t_{k} \leq t \leq t_{k+1}$

$$
X_{n}^{(h)}(t)=X_{n}^{(h)}\left(t_{k}\right)+2 \beta X_{n}^{(h)}\left(t_{k}\right)\left(t-t_{k}\right)+\int_{t_{k}}^{t} \delta_{u} d u+g^{\prime}\left(X_{n}^{(h)}\left(t_{k}\right)\right)\left(B_{t}-B_{t_{k}}\right)
$$

converges on $\llbracket 0, \sigma_{h} \wedge T \rrbracket$ towards the unique strong solution of

$$
d X_{s}^{(h)}=\left(2 \beta X_{s}^{(h)}+\delta_{s}\right) d s+g^{\prime}\left(X_{s}^{(h)}\right) d B_{s}
$$

in the sense that $\sup _{0 \leq s \leq t}\left|X_{n}^{(h)}(s)-X^{(h)}(s)\right|$ converges in probability to zero for each $t \leq T \wedge \sigma_{h}$.

In order to prove the $L^{1}$-sup convergence on $\llbracket 0, \sigma_{h} \wedge T \rrbracket$, one need to check that $\sup _{0 \leq s \leq t}\left|X_{n}^{(h)}(s)-X^{(h)}(s)\right|$ is uniformly integrable in $n$. This is straightforward by using Itô's lemma, Cauchy-Schwarz' inequality and the BurkholderDavis-Gundy inequality and therefore, the calculations are omitted. 
We conclude that $\lim _{n \rightarrow \infty} \mathbb{E}\left[\sup _{0 \leq s \leq t}\left|X_{n}^{(h)}(s)-X^{(h)}(s)\right|\right]=0$ for all $t \leq$ $T \wedge \sigma_{h}$. But on $\llbracket 0, \sigma_{h} \wedge T \rrbracket$, all solutions $X^{(m)}$ with $m \geq h$ have to be indistinguishable by uniqueness. Since $\mathbb{P}\left[\sup _{0 \leq t \leq T} X_{t} \geq h\right]$ converges to zero for $h$ going to infinity, $\bigcup \llbracket 0, \sigma_{h} \wedge T \rrbracket=\llbracket 0, T \rrbracket$ and on $\llbracket 0, T \rrbracket$, the Euler scheme

$$
X_{n}(t)=X_{n}(0)+\int_{0}^{t} \delta_{u} d u+\int_{0}^{t} 2 \beta X_{n}\left(\eta_{n}(u)\right) d u+\int_{0}^{t} g^{\prime}\left(X_{n}\left(\eta_{n}(u)\right)\right) d B_{u}
$$

converges in $L^{1}$-supnorm towards the unique solution of (2), namely

$$
d X_{s}=\left(2 \beta X_{s}+\delta_{s}\right) d s+g\left(X_{s}\right) d B_{s} .
$$

It is now easy to see that the Euler discretization scheme

$$
X_{n}(t)=X_{n}(0)+\int_{0}^{t} \delta\left(\eta_{n}(u)\right) d u+\int_{0}^{t} 2 \beta X_{n}\left(\eta_{n}(u)\right) d u+\int_{0}^{t} g^{\prime}\left(X_{n}\left(\eta_{n}(u)\right)\right) d B_{u}
$$

also converges towards the solution of (2) since

$$
\mathbb{E}\left[\sup _{0 \leq t \leq T}\left|\int_{0}^{t} \delta_{u} d u-\int_{0}^{t} \delta\left(\eta_{n}(u)\right) d u\right|\right] \leq \int_{0}^{T} \mathbb{E}\left[\left|\delta_{u}-\delta\left(\eta_{n}(u)\right)\right|\right] d u,
$$

which converges to zero as $\left(\delta_{u}\right)_{u \geq 0}$ is a uniformly integrable family.

Let us now look at the general case with the local assumption $\sup _{u} \delta_{u} \in L^{1}$. We define the sequence of stopping times $\left(\tau_{p}\right)_{p \geq 1}$, by $\tau_{p}=\inf \left\{t \mid \int_{0}^{t} \delta_{u} d u \geq p\right\}$ and we denote $\delta_{u} \mathbb{1}_{\llbracket 0, \tau_{p} \rrbracket}$ by $\delta_{u}^{(p)}$. From these definitions follows that

$$
\mathbb{E}\left[\left(\int_{0}^{T} \delta_{u}^{(p)} d u\right)^{2}\right] \leq p^{2}
$$

Therefore, we can apply the first part of the proof in case of the stochastic differential equation

$$
d X_{s}^{(p)}=\left(2 \beta X_{s}^{(p)}+\delta_{s}^{(p)}\right) d s+g\left(X_{s}^{(p)}\right) d B_{s}
$$

to obtain that the Euler scheme

$X_{n}^{(p)}(t)=X_{n}^{(p)}(0)+\int_{0}^{t} \delta^{(p)}\left(\eta_{n}(u)\right) d u+\int_{0}^{t} 2 \beta X_{n}^{(p)}\left(\eta_{n}(u)\right) d u+\int_{0}^{t} g^{\prime}\left(X_{n}^{(p)}\left(\eta_{n}(u)\right)\right) d u$

converges in $L^{1}$-supnorm towards the unique solution of (3). On $\llbracket 0, \tau_{p} \rrbracket$, all $X^{(k)}$ with $k \geq p$ are indistinguishable because of the uniqueness of the solution of the stochastic differential equation $(2)$. Since $\llbracket 0, T \rrbracket \subset \bigcup \llbracket 0, \tau_{p} \rrbracket$, the Euler scheme 
converges almost everywhere to the unique solution of (2).

By the same reasoning, we find that for each time $l \geq 0$, the scheme is convergent to the solution of the stochastic differential equation (2) on the $\llbracket 0, l \rrbracket$, denoted by $X^{(l)}$. By uniqueness, the solutions $\left(X^{(l)}\right)_{l \geq 0}$ have to be extensions of each other.

q.e.d.

Remark that by localization, it is easy to extend this result by allowing a volatility function defined by $|g(x)|=k x^{\alpha}$ with $k$ a constant and $\alpha$ a real number between $1 / 2$ and 1 .

\subsection{Convergence in $\mathcal{H}^{1}$-norm}

We now turn to the convergence of the discretization scheme in $\mathcal{H}^{1}$-norm. We check the $\mathcal{H}^{1}$-convergence since the space of special semimartingales with finite $\mathcal{H}^{p}$-norm appears more and more often in mathematical finance, for example in arbitrage theory (e.g. Föllmer-Schweizer ${ }^{19}$, Ansel-Stricker ${ }^{20}$, DelbaenSchachermayer ${ }^{21,22}$ ). Besides, Protter ${ }^{12}$ studied the $\mathcal{H}^{p}$ perturbations of semimartingale differentials and showed that this approach is suited to obtain almost sure stability of solutions, a problem that was noticed by Wong and Zakai ${ }^{23}$.

Let us recall from Protter $^{12}$ that for a continuous semimartingale $Z$ with $Z_{0}=0$, the $\mathcal{H}^{1}$-norm is equivalent with the norm

$$
j_{1}(N, A)=\left\|[N, N]_{\infty}^{1 / 2}+\int_{0}^{\infty}\left|d A_{s}\right|\right\|_{L^{1}}
$$

where $Z=N+A$ is the decomposition of $Z$ in its martingale part $N$ and its predictable part $A$ with paths of finite variation on compacts. If we assume $Z$ to be the difference $X_{n}-X_{n^{\prime}}$, then it is evident that $\left(X_{n}\right)_{n \geq 1}$ is a Cauchy sequence in the space of semimartingales with the $\mathcal{H}^{1}$-norm, which is complete. Since the $\mathcal{H}^{1}$-norm is finer than the $L^{1}$-supnorm, $\left(X_{n}\right)_{n \geq 1}$ also converges in $\mathcal{H}^{1}$-norm towards $X$, the solution of the stochastic differential equation.

\section{ORDER OF STRONG CONVERGENCE}

The results in the previous section also could be obtained by using Yamada's method. Although this method involves longer calculations, we give a sketch because it leads to immediate results about the order of strong convergence.

While the Euler approximation is one of the simplest time discrete approximations, it is in general not particularly efficient numerically. It might be useful to investigate other discretization schemes in case of stochastic differential equations with a drift term depending on a stochastic process with random 
correlation. In order to assess and compare different discretization schemes, one need to know the rates of strong convergence.

We recall from Kloeden-Platen ${ }^{16}$ that a time discrete approximation strongly converges with order $\nu$ at time $T$ if there exists a positive constant $C$, which does not depend on $\left\|\triangle_{n}\right\|$, and an $\varepsilon>0$ such that $\mathbb{E}\left(\left|X(T)-X_{n}(T)\right|\right) \leq C\left\|\triangle_{n}\right\|^{\nu}$ for each $\left\|\triangle_{n}\right\| \leq \varepsilon$. We now show that it is easy to check that the Euler scheme strongly converges with order $\nu=0.5$ at time $T$ as soon as the stochastic drift process $\delta$ is fulfilling the hypotheses of the previous section and is such that there exists a constant $k$, which does not depend on $\left\|\triangle_{n}\right\|$, so that

$$
\mathbb{E}\left[\left|\int_{0}^{t}\left(\delta\left(\eta_{n}(u)\right)-\delta(u)\right) d u\right|\right] \leq k\left\|\triangle_{n}\right\|^{1 / 2}
$$

for all $t \in[0, T]$ and all $\left\|\triangle_{n}\right\| \leq \varepsilon$. We maintain the notation of the previous section.

First, we remark that it is easy to establish an explicit bound for $\mathbb{E}\left[\left|X_{n}\left(\eta_{n}(t)\right)\right|\right]$ with $t_{k} \leq t \leq t_{k+1}$, by using Gronwall's inequality, namely

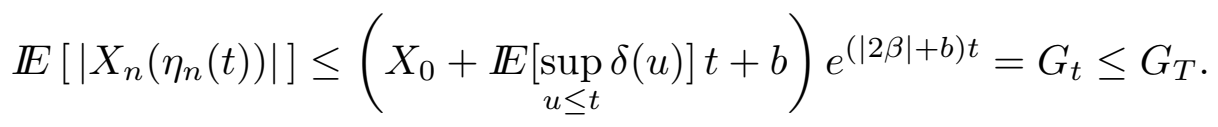

This upperbound is independent of $n$ and $t$. by

¿From this inequality it follows that $\left\|X_{n}(t)-X_{n}\left(\eta_{n}(t)\right)\right\|_{1}$ is bounded above

$$
|2 \beta| G_{T}\left\|\triangle_{n}\right\|+\mathbb{E}\left[\sup _{u \leq T} \delta(u)\right]\left\|\triangle_{n}\right\|+b \sqrt{G_{T}} \sqrt{\left\|\triangle_{n}\right\|},
$$

which we denote by $H_{T}(n)$. This bound is independent of $t$ and converges to zero for $n$ tending to infinity with order $o\left(\left\|\triangle_{n}\right\|^{1 / 2}\right)$.

We use these intermediate results to prove that $\left(X_{n}\right)_{n \geq 1}$ is a Cauchy sequence in $L^{1}([0, T] \times \Omega)$. Let us introduce a sequence of functions like in Yamada's ${ }^{14}$ paper (see e.g. Karatzas-Shreve ${ }^{5}$ p. 291). We choose a strictly decreasing sequence $\left\{a_{n}\right\}_{n=0}^{\infty} \subset(0,1]$ with $a_{0}=1$ such that $\lim _{n \rightarrow \infty} a_{n}=0$ and $\int_{a_{n}}^{a_{n-1}} \frac{d u}{b^{2} u}=n$ for every $n \geq 1$. For each $n \geq 1$, there exists a continuous function $\rho_{n}$ on $\mathbb{R}$ with support in $\left(a_{n-1}, a_{n}\right)$ so that $0 \leq \rho_{n}(x) \leq \frac{2}{n b^{2} x}$ holds for every $x>0$ and $\int_{a_{n}}^{a_{n-1}} \rho_{n}(x) d x=1$. Then the function

$$
\varphi_{m}(x)=\int_{0}^{|x|} \int_{0}^{y} \rho_{n}(u) d u d y \text { for all } x \in \mathbb{R}
$$

is even and twice continuously differentiable, with $\left|\varphi_{m}^{\prime}(x)\right| \leq 1$ and for $x \in \mathbb{R}$ $\lim _{n \rightarrow \infty} \varphi_{n}(x)=|x|$, where the sequence $\left\{\varphi_{n}\right\}_{n=1}^{\infty}$ is nondecreasing. Furthermore, remark that $|u|-a_{m-1} \leq \varphi_{m}(u)$. Consequently, we have that $\left|X_{n}(t)-X_{n^{\prime}}(t)\right| \leq a_{m-1}+\varphi_{m}\left(X_{n}(t)-X_{n^{\prime}}(t)\right)$. We use this property to estimate the $L^{1}$-norm $\left\|X_{n}(t)-X_{n^{\prime}}(t)\right\|_{1}$. 
Indeed, applying Itô's lemma, one finds the stochastic differential equation of $\varphi_{m}\left(X_{n}(t)-X_{n^{\prime}}(t)\right)$ and taking expectations, one obtains (after some long calculations) that

$$
\begin{aligned}
& \mathbb{E}\left[\left|X_{n}(t)-X_{n^{\prime}}(t)\right|\right] \\
& \leq \quad a_{m-1}+\mathbb{E}\left[\varphi_{m}\left(X_{n}(t)-X_{n^{\prime}}(t)\right)\right] \\
& \leq \quad a_{m-1}+\frac{3 T}{m}+\left(H_{T}(n)+H_{T}\left(n^{\prime}\right)\right)\left(\frac{3}{2}\left\|\varphi_{m}^{\prime \prime}\right\| b^{2}+|2 \beta|\right) T \\
& \quad+\mathbb{E}\left[|| \int_{0}^{t}\left(\delta\left(\eta_{n}(u)\right)-\delta\left(\eta_{n^{\prime}}(u)\right)\right) d u \mid\right]
\end{aligned}
$$

For a given $\left\|\triangle_{n}\right\| \leq \varepsilon, m$ can be chosen such that $0<a_{m-1}<\left\|\triangle_{n}\right\|^{1 / 2}$ and $\frac{3 T}{m}<\left\|\triangle_{n}\right\|^{1 / 2}$. For this fixed $m,\left\|\varphi_{m}^{\prime \prime}\right\|$ is known to be bounded and therefore, we can choose an $n_{0}$ such that the third term remains smaller than $C\left\|\triangle_{n}\right\|^{1 / 2}$ with $C$ a constant. Since by hypothesis,

$$
\mathbb{E}\left[\left|\int_{0}^{t}\left(\delta\left(\eta_{n}(u)\right)-\delta(u)\right) d u\right|\right] \leq k\left\|\triangle_{n}\right\|^{1 / 2},
$$

we conclude that the Euler scheme strongly converges with order $\nu=1 / 2$ at time $t \leq T$.

q.e.d.

It would be interesting to consider higher order schemes in case of stochastic differential equations depending on a stochastic process with random correlation and to compare them numerically through computer experiments.

\section{ACKNOWLEDGEMENTS}

The authors would like to thank the referees of this paper for their helpful comments.

\section{References}

[1] J.C. Cox, J.E. Ingersoll and S.A. Ross, 'A theory of the term structure of interest rates', Econometrica, 53, 385-407 (1985).

[2] G. Deelstra and F. Delbaen, 'Long-term returns in stochastic interest rate models', Insurance: Mathematics and Economics, 17, 163-169 (1995).

[3] G. Deelstra and F. Delbaen, 'Long-term returns in stochastic interest rate models: Convergence in law', Stochastics and Stochastics Reports, 55, 253277 (1995). 
[4] J. Jacod, 'Une Condition d'Existence et d'Unicité pour les Solutions fortes d'Équations Différentielles Stochastiques', Stochastics, Vol.4, 23-28, (1980).

[5] I. Karatzas and S.E. Shreve, Brownian Motion and Stochastic Calculus, Springer-Verlag, New York, Berlin, Heidelberg, 1988.

[6] D. Revuz and M. Yor, Continuous Martingales and Brownian Motion, Springer-Verlag Berlin, Heidelberg, New York, 1991.

[7] G. Deelstra, 'Long-term returns in stochastic interest rate models', Ph.D. thesis, Free University of Brussels, 1995.

[8] I.I. Gihman and A.V. Skorohod, Stochastic differential équations, SpringerVerlag, Berlin, Heidelberg, New York, 1972.

[9] V. Bally, 'Approximation of the solution of SDE's, I: $L^{p}$ convergence', Stochastics and Stochastics Reports, 28, 209-246 (1989).

[10] V. Bally, 'Approximation of the solution of SDE's, II: Strong convergence', Stochastics and Stochastics Reports, 28, 357-385 (1989).

[11] T. Kurtz and P. Protter, 'Wong-Zakai Corrections, Random Evolutions, and Simulation Schemes for SDE's', in Stochastic Analysis, Mayer-Wolf, Merzbach, Schwarz eds, Academic Press, 1991, pp. 331-346.

[12] P. Protter, ' $\mathcal{H}^{p}$ Stability of Solutions of Stochastic Differential Equations', Z. Wahrscheinlichkeitstheorie verw. Gebiete, 44, 337-352 (1978).

[13] P. Protter, Stochastic Integration and Differential Equations, SpringerVerlag, Berlin, Heidelberg, New York, 1990.

[14] T. Yamada, 'Sur une construction des solutions d'équations différentielles stochastiques dans le cas non-Lipschitzien', in Séminaire de Probabilité XII, LNM 649, Springer-Verlag, Berlin, Heidelberg, New York, 1978, pp.114131.

[15] R. Bucy and P. Joseph, Filtering for Stochastic Processes with Applications to Guidance, Chelsea Publishing Company, New York, N.Y., 1987.

[16] P.E. Kloeden and E. Platen, Numerical Solution of Stochastic Differential Equations, Springer-Verlag, Berlin, Heidelberg, New York, 1992.

[17] N.J. Cutland, E. Kopp and W. Willinger, 'From Discrete to Continuous Financial Models: New Convergence Results for Option Pricing', Mathematical Finance, 3 (2), 101-123 (1993).

[18] T. Kurtz and P. Protter, 'Weak Limit Theorems for Stochastic Integrals and Stochastic Differential Equations', The Annals of Probability, 19 (3), 1991, 1035-1070. 
[19] H. Föllmer and M. Schweizer, 'Hedging of Contingent Claims under Incomplete Information', in Davis M.H.A. and Elliott R.J. eds.: Applied Stochastic Analysis, Stochastic Monographs 5, London, New york, Gordon and Breach, 1991, pp. 389-414.

[20] J.P. Ansel and C. Stricker, 'Lois de martingale, densités et décomposition de Föllmer-Schweizer', Annales de l'Institut Henri Poincaré, 28, 375-392 (1993).

[21] F. Delbaen and W. Schachermayer, 'A General Version of the Fundamental Theorem of Asset Pricing', Mathematische Annalen, 300, 463-520 (1994).

[22] F. Delbaen and W. Schachermayer, 'The No-Arbitrage Property under a Change of Numéraire', Stochastics and Stochastic Reports, 53, 213-226 (1995).

[23] E. Wong and M. Zakai, 'On the convergence of ordinary integrals to stochastic integrals', Ann. Math. Statist., 36, 1560-1564 (1965). 